\title{
Investigation of snowboard stiffness and camber characteristics for different riding styles
}

\author{
A. Subic $\cdot$ P. Clifton $\cdot$ J. Beneyto-Ferre $\cdot$ \\ A. LeFlohic $\cdot$ Y. Sato $\cdot$ V. Pichon
}

Published online: 26 November 2008

(c) The Author(s) 2008. This article is published with open access at Springerlink.com

\begin{abstract}
Previous research has indicated that the flex pattern and camber of a snowboard are crucial to its perceived "feel", or the physical and psychological feedback given to the rider whilst snowboarding. These features are the primary cause of variation in snowboard performance for different riding styles. Consequently, this article deals with the identification of stiffness and camber characteristics for freestyle, freeride and versatile test boards, and their statistical correlation to a comprehensive list of qualitative feel-based performance requirements. It has been determined that the test boards spanning the major styles all possessed similar bending profiles, that were highly representative of each snowboard's respective thickness distribution. The torsional stiffness curves however appeared to be driven by the composite architecture used in construction. Unsurprisingly, the freeride test board showed the greatest level of overall stiffness. The versatile board exhibited the greatest fluctuation in bending stiffness along the chord, whereas the freestyle profile was far more even throughout, with less variation from tip to tail. All of the subjective performance parameters except forgiveness showed positive associations to the body stiffness and camber, with manoeuvrability exhibiting the strongest correlations. The forgiveness showed the exact opposite trend, implying that higher levels of flex and less camber promotes a forgiving snowboard.
\end{abstract}

\footnotetext{
A. Subic $(\bowtie) \cdot$ P. Clifton · J. Beneyto-Ferre · A. LeFlohic ·

Y. Sato - V. Pichon

School of Aerospace, Mechanical and Manufacturing Engineering, RMIT University, Melbourne, Australia e-mail: Aleksandar.Subic@ @rmit.edu.au
}

Keywords Snowboards · Stiffness tests - Correlation · Subjective performance ratings

\section{Introduction}

Modern snowboard design is dictated predominantly by the desired application or style of the ride, with boards generally falling under one of two headers; freestyle (park and trick-based) or freeride (all-mountain). Certain boards are also considered 'versatile' (do not fall under either major style heading), and are designed to bridge the gap between the two major styles. A third, less popular race-specific category also exists, that of freecarving or alpine. Manufacturers currently spend significant time and money trialling new designs, relying heavily on the feedback of professional riders in attempting to design-in the "feel", being the physical and psychological feedback given to the rider whilst snowboarding, as well as optimise the performance of the board. A systematic user-centred design procedure could provide the intelligence required to alleviate the trial and error approach, resulting in higher customer satisfaction as well as cost and time savings.

The Snowboard Research Group at RMIT University in Melbourne set out to fully characterise the feel of snowboards for the two main riding styles. By correlating subjective evaluations to objective laboratory and field based data, the relevant matrices of parameters leading to the desired feel of the board can be determined. Preliminary research [8] has indicated that the flex pattern (bending and torsional stiffness distributions) and camber (maximum height of the running surface) of the snowboard are crucial to its overall feel and response. Furthermore, they result in different snowboard performance for different riding styles. 
Table 1 Subjective parameter definitions

\begin{tabular}{ll}
\hline Subjective parameter & Definition \\
\hline Stability & How stable the rider feels on the board \\
Manoeuvrability & How easily the board responds to rider inputs \\
Accuracy & The precision of board movement in response to rider input \\
Edge grip & The level of grip exhibited during turns \\
Feedback & The amount of stress felt on the rider's body including the effects of board chatter \\
Forgiveness & The tolerance of the board to errors from the rider \\
Speed & The gliding speed of the board compared to other boards of similar length \\
Liveliness & The level of 'pop' or spring in the board when performing a jump \\
Transition & How easily the board flows from edge to edge \\
smoothness &
\end{tabular}

Other studies have also highlighted the importance of stiffness to snowboard performance and furthermore have touched on correlative aspects. Buckingham and Blackford [2] sampled the bending and torsional stiffness of four snowboards and loosely correlated the data to qualitative on-slope performance evaluations of general feel, as well as perceived overall and torsional stiffness. Buffinton et al. [3] collected a large amount of quantitative data in the laboratory and on-snow for three snowboards, but again only undertook a non-statistical correlation of natural frequencies and damping ratios to vague subjective manufacturer descriptions. More extensive studies [4, 5] on the correlation between subjective performance and mechanical properties of skis were utilised in this research as reference points.

This article deals with the identification of stiffness and camber characteristics for one representative board from each of the freeride, freestyle and versatile riding styles, and their correlation to the qualitative feel based performance requirements contained in Table 1 . These parameters were determined from prior research [8] to comprehensively characterise a snowboard's feel and performance from the standpoint of the rider. The three test boards were selected based on a combination of overall popularity from the initial survey process (see below), and manufacturer/seller descriptions of their location within the stiffness spectrum. A highly stiff $159 \mathrm{~cm}$ freeride board and conversely a very flexible $154 \mathrm{~cm}$ pure freestyle board were chosen to represent the end points of the spectrum, whilst a third popular $157 \mathrm{~cm}$ versatile board was selected as an intermediary. The camber was not considered during the selection process.

\section{The importance of stiffness and camber in snowboard design}

A comprehensive benchmarking analysis has been undertaken to identify the key snowboard design parameters and potential innovation opportunities [8]. The analysis used the quality function deployment (QFD) [10] method to evaluate relevant customer requirements and relate them to objective technical attributes of selected boards for both major riding styles. Additionally, key design drivers were used in conjunction with market opportunity mapping $[7,9]$ to identify potential gaps in the snowboard equipment market.

The qualitative data relating to customer requirements was obtained through a variety of online and on-snow surveys and interviews. Participants in an initial mass circulated online survey (115 respondents) were asked to rate and comment on their current board using an extensive list of subjective parameters, spanning all facets of riding. A refined parameter list (Table 1) was obtained through subsequent interviews with selected focus groups (nine respondents), with importance values and ideal levels determined for both freestyle and freeride boards.

On-snow testing and interviews using the three high quality test boards were also conducted to obtain subjective ratings with a strong statistical basis, and determine the interrelationships for each of the qualitative parameters. Eight professionals (snowboard instructors) were employed to ride a set slalom course that examined each board's ability to undertake turns of varying difficulty, as well as the performance of jumps and tricks. After performing two runs, riders were interviewed as to the levels of each subjective parameter present in the test board (on a scale of 1-10), and whether these levels were, in their opinion, toohigh, too-low, or optimal. If assessed as sub-optimal, the riders were asked to further estimate the margin by which the subjective parameters varied from optimal levels. To reduce variables present in the testing, all riders used the same make, model and size binding, which required at least a size 9 (US) foot. This also ensured the variance in rider mass was minimised. All tests were undertaken over a two day period, where the temperature fluctuated between -1 and $3^{\circ} \mathrm{C}$. The results of the field tests are displayed in Table 2, containing average values of each subjective parameter for all three test boards. 
Table 2 Subjective correlation data

\begin{tabular}{llll}
\hline & Freeride & Versatile & Freestyle \\
\hline Stability & 9.0 & 7.0 & 6.2 \\
Manoeuvrability & 8.0 & 7.2 & 7.8 \\
Accuracy & 8.9 & 7.8 & 7.1 \\
Edge grip & 9.5 & 7.3 & 6.9 \\
Feedback & 4.4 & 4.3 & 5.1 \\
Forgiveness & 5.4 & 6.8 & 6.5 \\
Speed & 8.5 & 8.1 & 5.1 \\
Liveliness & 6.7 & 6.6 & 7.9 \\
Transition smoothness & 8.8 & 8.7 & 6.0 \\
\hline
\end{tabular}

The objective technical attributes used in the QFD analysis were based primarily on the ASTM Standard F1107-1995-Standard Terminology Relating to Snowboarding [1], although several other quantifiable parameters relating to material properties were defined to cover all relevant aspects of the snowboard design. All of the parameters were measured in the laboratory or obtained from published data sheets. The QFD methodology used various correlations between the subjective and objective parameters to identify the key objective design features associated with the desired feel of the board. It highlighted the bending stiffness distribution as the overall key objective parameter. For full details regarding both the subjective analysis and objective data collected for the three test boards, refer to [8].

A comprehensive gap analysis [7,9] was also completed to identify possible design innovation and product development opportunities for modern snowboards. Using the ratings from the first online survey of board models manufactured between 2004 and 2007, the snowboard's cumulative performance under the prescribed qualitative headers was plotted against its published style, within a range between pure freeride and pure freestyle. The performance measure for each model was calculated using the weighted average of ratings, compared to the ideal levels of each parameter within the prescribed style, as follows:

$P=\frac{1}{\frac{1}{9} \sum_{i=1}^{9}\left(W_{i} \times\left|R_{i}-I_{i}\right|\right)}$

where $P$ is performance, $W_{i}$ is the importance weight for the $i$ th parameter, $R_{i}$ is the Board rating for the $i$ th parameter and $I_{i}$ is the ideal level of the $i^{\text {th }}$ parameter. The resulting market opportunity map showed that there were practically no high performing versatile boards present, at odds with the desires of modern snowboarders from the various surveys and interviews. This confirmed that a gap in the current snowboard marketplace exists, which provides potential design innovation opportunities for high performing, versatile snowboards.
In order to realise the identified design innovation opportunity, it was important to identify the objective design parameters that affect the versatility of snowboards. A versatility value was formulated as a measure of the extent a variation in an objective design parameter will drive the feel and performance from freestyle to freeride or vice versa. It was defined as the product of the average relative importance weight from the QFD chart and the normalised range of objective technical assessment data between the three test boards:

$V=\frac{W_{\mathrm{FS}}+W_{\mathrm{FR}}}{2} \times \frac{O_{\max }-O_{\min }}{O_{\max }}$

where $V$ is versatility value, $W_{\mathrm{FS}}$ is freestyle importance weight, $W_{\mathrm{FR}}$ is freeride importance weight, $O_{0}$ is maximum objective parameter value and $O_{\min }$ is minimum objective parameter value. It was noted that several features appeared to be crucial to the versatility of a modern snowboard. The self-weighted camber, bending/torsional stiffness in the body and the body stiffness/weight ratio all possessed exceptionally high versatility values, however the low value of the mass parameter indicated that stiffness was of key concern as the mass did not vary to any significant extent between the test boards. Furthermore, the source of the high values differed between the three design features. The bending stiffness value was primarily the result of a very high relative importance weight from the QFD analysis, indicating its significance to the overall feel for both styles. The camber and torsional stiffness values however were due to large data ranges between the test boards, and the relative importance values were notably lower. Overall, the benchmarking indicated varying the bending and torsional stiffness distributions and the camber would appear to be the key approach to altering the feel and performance of a snowboard across the major riding styles.

\section{Experimental investigation of stiffness and camber characteristics}

Given the determined importance of bending and torsional stiffness distributions plus camber to the overall feel of snowboards, as well as driving the difference between the major riding styles, a thorough investigation into the bending and torsional characteristics of the three test boards was undertaken. The results should assist greatly in the generation of a versatile design characterised by optimal feel, as well as provide further insight into snowboard designs in general.

The procedures for the tests were based primarily on ISO Standard 5902: Alpine Skis-Determination of the Elastic Properties [6] although various modifications were made to the procedures in order to apply them to snowboards. Most changes from the tests prescribed in the 
standard were a result of the differing geometry between snowboards and skis, requiring alternate testing dimensions as well as the application of greater forces and moments on the test boards to generate the requisite levels of bending and torsion. Firstly, as each ski only possesses one binding as opposed to the dual binding system present on snowboards, the location of clamping during the tests had to be modified. Secondly, since only the basic testing methods were covered by the standard, a testing rig had to be designed and manufactured (see below for details). Finally, the standard only gave procedures for calculating spring constants, with units of $\mathrm{N} / \mathrm{mm}$ and $\mathrm{N} \mathrm{m} /{ }^{\circ}$ for bending and torsion respectively, so further transformation of the data was required to enable the calculation of relevant stiffness values. This transformation was based around the work of [4] that sets out the calculation of bending and torsional stiffness from basic deflection and angular deformation measurements. The combination of both of these approaches here allowed the determination of bending and torsional stiffness curves for all three test boards.

Note that as per the ASTM Standard F1107-1995Standard Terminology Relating to Snowboarding [1], the shovel and heel of the snowboard are the sections from the forward and aft base contact points to the tip and tail respectively, with the snowboard unweighted on a plane surface. The body of a snowboard has been defined by the authors to lie between the heel and shovel.

\section{Bending stiffness}

The bending stiffness of the three test boards was calculated using the following basic formula [4]:

$E I=\frac{M}{f^{\prime \prime}}$

where $E I$ is the bending stiffness $\left(\mathrm{N} \mathrm{m}^{2}\right), M$ is the applied moment $(\mathrm{N} \mathrm{m})$ and $f^{\prime \prime}$ is the curvature of the snowboard $\left(\mathrm{m}^{-1}\right)$. The apparatus designed for the tests based on the descriptions given in the standard is pictorially shown in Figs. 1-3 below. The test rig displayed in Fig. 1 consists of a 1,500 mm long C-channel base, two adjustable supports with $20 \mathrm{~mm}$ diameter rollers (capable of supporting the entire width of all three snowboards from the tip and tail, respectively), and finally a load $(F)$ application device consisting of two $20 \mathrm{~mm}$ diameter rollers supporting two $16 \mathrm{~kg}$ masses via hooks. This particular setup allowed the calculation of the bending stiffness in the body section of the test boards. To determine the bending stiffness at the heel and shovel of the snowboards, a different setup was required, as shown in Fig. 2. For these particular tests the forebody or aftbody of the board was clamped using sets of $40 \mathrm{~mm}$ wide metal plates, and the

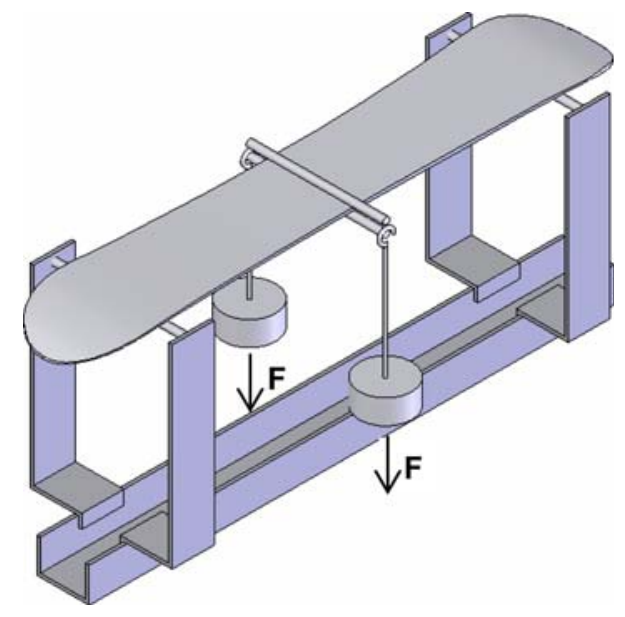

Fig. 1 Bending tests rig

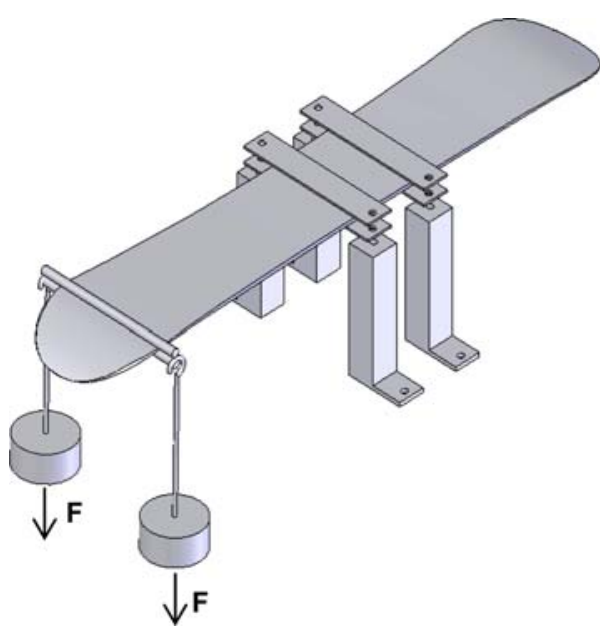

Fig. 2 Alternate setup

board was deflected using $22 \mathrm{~kg}$ of total mass $100 \mathrm{~mm}$ from the tip/tail.

Figure 3 shows the apparatus used to calculate the curvature of the snowboard, consisting of a $20 \mathrm{~mm}$ comparator positioned centrally in a $200 \mathrm{~mm} \mathrm{C}$-section. The device allowed accurate measurement of the localised relative deflection $(x)$ at $50 \mathrm{~mm}$ intervals along the chord, and thus calculation of the curvature using the following simple geometry (shown in Fig. 4).

Using Pythagoras theorem:

$R^{2}=\left(\frac{L}{2}\right)^{2}+(R-x)^{2}$

when $R \gg x$,

$R \approx \frac{L^{2}}{8 x}$

Thus the curvature $(1 / R)$ could easily be determined. The combined results of the bending tests on all three test 


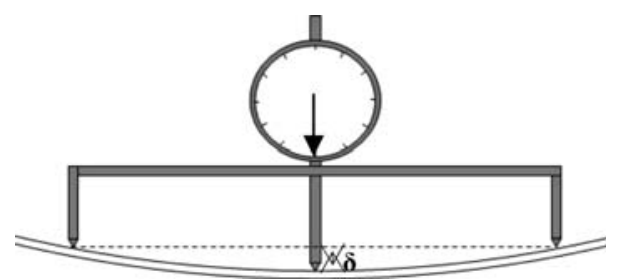

Fig. 3 Curvature measurement device

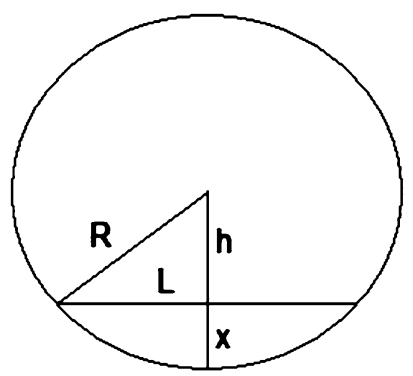

Fig. 4 Curvature geometry

boards are shown graphically in Fig. 5. Due to the assumptions and simplifications inherent in the calculation of the curvature, together with the usual human measurement errors, the maximum expected error in the final bending values was calculated at approximately $5 \%$.

\section{Torsional stiffness}

The torsional stiffness profiles were obtained using the following formula [4]:
$G J=\frac{T d}{\phi}$

where $G J$ represents the torsional stiffness $\left(\mathrm{N} \mathrm{m}^{2}\right), T$ is the applied torque $(\mathrm{N} \mathrm{m}), \phi$ is the angular deformation (rad) and $d$ is the length of the area under consideration (m). Given that the board materials were non-homogenous, the measurements were made upon four portions of the board approximately $200 \mathrm{~mm}$ long. This length was chosen as although it should ideally have been as small as possible, due to angle measurement accuracy considerations it was considered appropriate. It was also a slight departure in procedure from the method of [4], who used the total length from the clamping device, but resulted in higher levels of accuracy as the original formula was for a homogenous beam. Figure 6 below displays the approximate segmenting of the board for the torsional tests. Using the same rig as per the forebody and aftbody bending tests to clamp the board, the portion under consideration was twisted using a dual system, comprising of a hanging mass on one side of the snowboard section, and a mass pulling the board upward via a pulley and flagstaff on the opposing side. This setup is shown in Fig. 7 below. Note that the masses applied to each test board varied between 23 and $11 \mathrm{~kg}$, to ensure adequate angular displacement without straying into the plastic deformation zone.

The board was clamped in four separate configurations for the tests. Firstly, the basic forebody/aftbody tests were conducted, whereby the board was clamped along the centreline, with the rollers positioned $170 \mathrm{~mm}$ from the tip/ tail (as per Fig. 7). Secondly, in order to simulate the twist generated on the body section of each snowboard by the
Fig. 5 Bending stiffness tests results

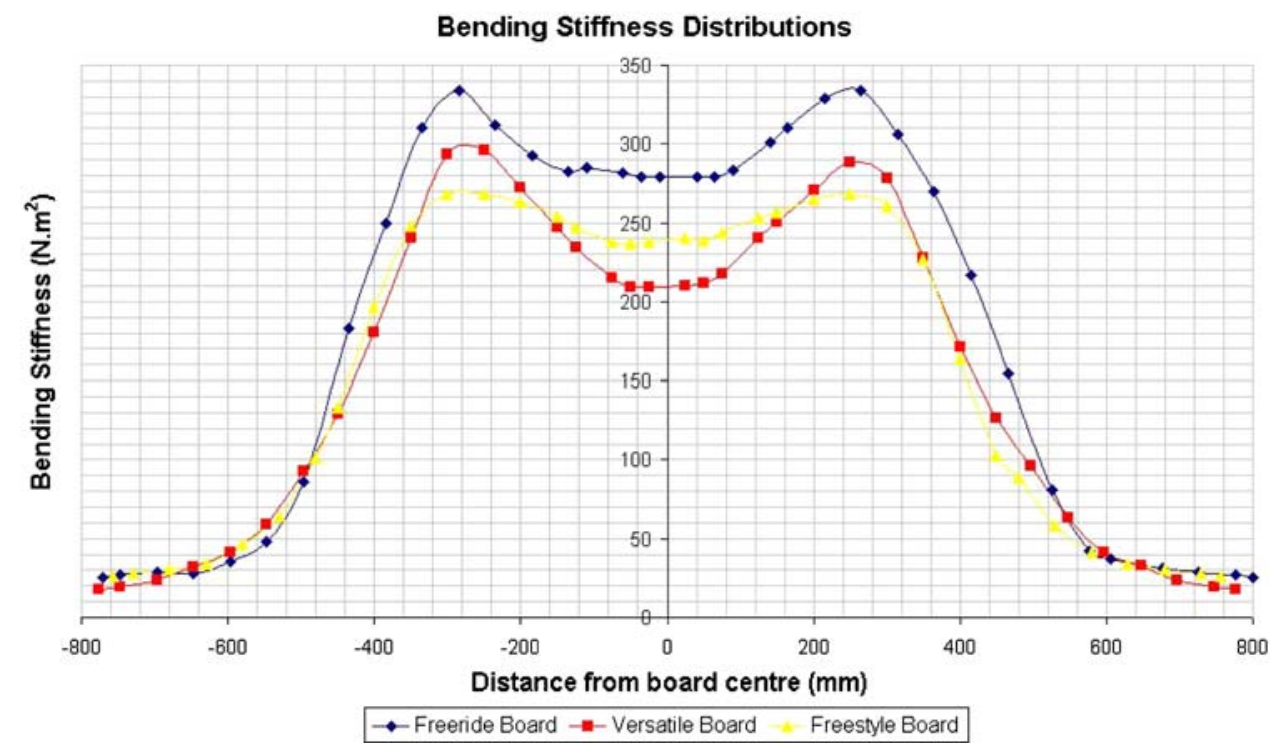




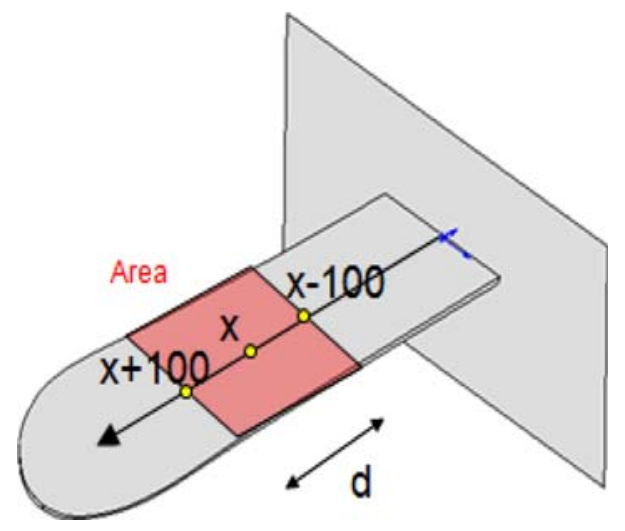

Fig. 6 Board measurement sections

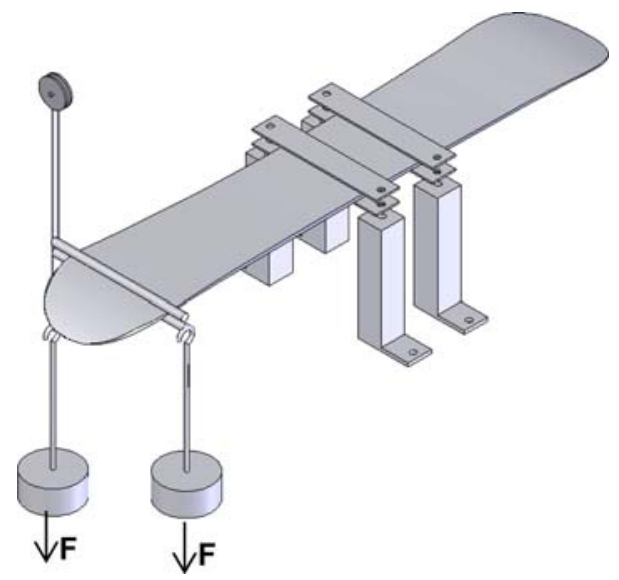

Fig. 7 Torsion tests rig

rider, the board was clamped at the forward binding location, with the rollers positioned on the aft binding inserts. This test was repeated with the opposing setup, with both tests utilising a test section of the stance width plus $100 \mathrm{~mm}$. The second set of tests was again a departure from both the standard and the work of [4], in order to highlight the particular response of the body section of each test board, determined to be crucial from prior research [8].

To calculate the resulting angular deformation along each test section, again a comparator (on a guided rail) was utilised to measure the vertical displacement of locations along the edge of the board (at $50 \mathrm{~mm}$ intervals), which were then converted into angles using the board width distribution and the following simple geometry (shown in Fig. 8):

Angular deformation:

$\phi=\arctan \left(\frac{b}{a}\right)$

where $a$ is the distance from the centreline of the board and $b$ is the vertical displacement. The resulting torsional stiffness profiles for the three test boards are displayed in

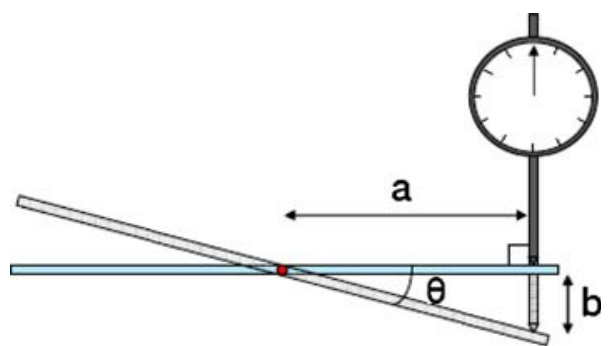

Fig. 8 Angular deformation measurement

Fig. 9 below. Note that unlike the bending profiles which were calculated from tip to tail, the difficulty in undertaking the torsional measurements on the curved heel and shovel sections meant that these areas of the board were neglected. However considering that torsional stress on the board is imparted almost solely by the riders' feet, the torsional stiffness in the body of the snowboard is paramount.

Compared to the bending profiles, the torsion characteristics possessed an almost equivalent maximum margin of error, estimated at approximately 5\%, primarily due to $200 \mathrm{~mm}$ segmenting length and angular measurement inaccuracy.

\section{Camber}

The camber of each test board was determined using a simple setup consisting of a comparator on a stand. It was calculated along the centreline of each snowboard by comparing readings when the board was pressed flat to a table surface and secondly when resting under only its own weight. Several measurements were taken and the results were averaged. Presented in millimeters with one decimal place (Table 3), the approximate error in the measurements was of the order of $1-2 \%$.

\section{Discussion of results}

The bending stiffness profiles in Fig. 5 showed a number of interesting trends across the style spectrum. All three boards possessed similar bending profiles, comprising a steep rise in stiffness from the tip and tail towards the centre, yet all featuring a substantial trough in the centre of the board. The wavelike profiles for all three snowboards were likely a result of the inherent geometry, with the sidecut largely responsible for the trough in the curve, and the steep rise towards the peaks due to a combination of the width and thickness distribution. The steel binding inserts would also likely have provided extra bending resistance around the locations of the peaks. Neglecting the trough in the curve, all three profiles were highly representative of 
Fig. 9 Torsional stiffness tests results
Torsional Stiffness Distributions

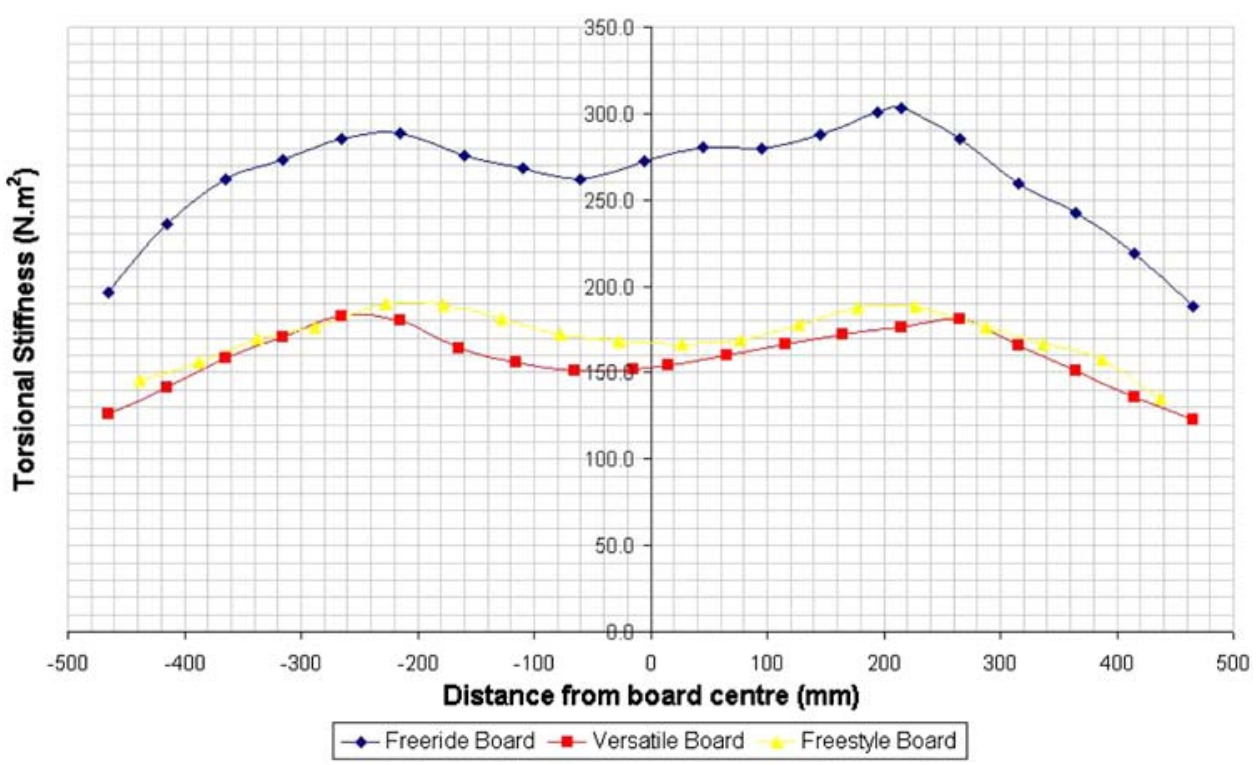

Table 3 Objective correlation data

\begin{tabular}{lrrr}
\hline & Freeride & Versatile & Freestyle \\
\hline Camber $(\mathrm{mm})$ & 8.1 & 4.4 & 7.9 \\
Shovel bending stiffness $\left(\mathrm{N} \mathrm{m}^{2}\right)$ & 30.4 & 27.3 & 32.0 \\
Body bending stiffness $\left(\mathrm{N} \mathrm{m}^{2}\right)$ & 234.4 & 196.5 & 200.5 \\
Heel bending stiffness $\left(\mathrm{N} \mathrm{m}^{2}\right)$ & 28.9 & 27.0 & 32.7 \\
Body torsional stiffness $\left(\mathrm{N} \mathrm{m}^{2}\right)$ & 266.0 & 160.3 & 172.6 \\
\hline
\end{tabular}

each snowboard's respective thickness distribution, and hence it can be postulated that the thickness of a snowboard at any location along its chord will drive its resulting bending stiffness. It is noted however that width, layer orientations and materials will also factor into the final bending stiffness profile.

The freeride test board unsurprisingly showed the greatest level of overall stiffness, with the peaks and troughs significantly higher than the remaining two test boards. Note also that the profile was not centred as the test board was asymmetric (longer shovel than heel). Surprisingly however, the versatile test board profile possessed higher peaks compared to the freestyle curve, yet a lower trough. Whether this was indicative of its versatility (being a combination of the other two curves) or was merely a coincidental result of the board's geometry requires further analysis. It was also noted that the freestyle profile was far more even throughout, with less drastic variation from tip to tail. This was not entirely unexpected given its intended trick riding application and the requirement of the board to have an even response.

The torsional profiles shown in Fig. 9 possessed similar trends to the preceding bending stiffness curves, with the characteristic wave profile apparent in all three test boards.
Again, the freeride test snowboard exhibited the greatest torsional stiffness, but in this case by a significant margin (approximately 150\%) unlike the previous bending curves. Considering that the geometry of the three test boards was not too dissimilar, and thus highly unlikely to have caused such a large torsional stiffness range, the materials and/or fibre orientation were likely to be the reason for the difference in torsional stiffness curves. The torsional profiles for the versatile and freestyle curves were very similar, but interestingly, the freestyle test board possessed a slightly greater torsional stiffness throughout the body. This was most likely a result of the board's relatively large waist width.

To consider the camber data (Table 3), interestingly the freeride board possessed the most camber, followed closely by the freestyle board. It was expected that given the trickbased nature of the style, the freestyle test board would have possessed the highest levels of camber, however when compared to the overall length of the board, the camber/ chord length ratios were essentially identical for the freeride and freestyle boards. Yet assessing the liveliness ratings for the two test boards, despite the similarity in relative camber levels, the freestyle board was rated as far more lively (Table 2). This could indicate that the high levels of bending stiffness present in the body section of the freeride board may have hindered its perception as a lively board, as greater effort by the rider is required to depress the cambered section of the board into the snow. Most surprising however, was that the versatile board exhibited the smallest level of camber, at just over $50 \%$ of the freeride value. This was probably due to the very low levels of bending stiffness present in the central portion of the body of the versatile test board, and furthermore, directly correlates with the board receiving the lowest rating for board liveliness. Comparison of the freeride 
and versatile boards indicates that they both are rated low for liveliness, although one has high bending stiffness whilst the other has a low value. This indicates the need for multiple parameter assessment and will be dealt with in the Correlation Analysis section below. It appears that the camber levels are strongly connected to the bending stiffness present in the very centre of the test boards, and further to achieve a certain level of camber in any final design, a threshold level of bending stiffness must first be present.

\section{Correlation analysis}

To provide further insight into the relationships between the bending and torsional stiffness characteristics (as well as camber) of snowboards and their performance, a statistical correlation analysis was performed between these objective parameters and the subjective performance ratings (Table 2) from the prior research. Since the stiffness data was in the form of continuous curves, for the correlation, integrated average values were calculated for each major portion of the three test boards, and are shown in Table 3 above. The table also displays the camber measurements used in the correlation analysis. Table 4 shows the resulting Spearman correlation coefficients (rounded to the nearest 0.5 ) for each relationship, on scale between +1 (increasing linear) and -1 (decreasing linear). These values were obtained by firstly converting each data set to ranks, then applying the following standard formula:

$\operatorname{Correl}(X, Y)=\frac{\sum(x-\bar{x})(y-\bar{y})}{\sqrt{\sum(x-\bar{x})^{2} \sum(y-\bar{y})^{2}}}$

where Correl $(X, Y)$ is the correlation coefficient, $x$ is the objective parameter rank, $y$ is the subjective parameter rank, whilst $\bar{x}$ and $\bar{y}$ are the respective sample rank means.

Table 4 Subjective-objective parameter correlations

\begin{tabular}{lrrrrr}
\hline & Camber & $\begin{array}{l}\text { Shovel } \\
\text { bending } \\
\text { stiffness } \\
\text { (avg) }\end{array}$ & $\begin{array}{l}\text { Body } \\
\text { bending } \\
\text { stiffness } \\
\text { (avg) }\end{array}$ & $\begin{array}{l}\text { Heel } \\
\text { bending } \\
\text { stiffness } \\
\text { (avg) }\end{array}$ & $\begin{array}{l}\text { Body } \\
\text { torsional } \\
\text { stiffness } \\
\text { (avg) }\end{array}$ \\
\hline Stability & 0.5 & -0.5 & 0.5 & -0.5 & 0.5 \\
Manoeuvrability & 1.0 & 0.5 & 1.0 & 0.5 & 1.0 \\
Accuracy & 0.5 & -0.5 & 0.5 & -0.5 & 0.5 \\
Edge grip & 0.5 & -0.5 & 0.5 & -0.5 & 0.5 \\
Feedback & 0.5 & 1.0 & 0.5 & 1.0 & 0.5 \\
Forgiveness & -1.0 & -0.5 & -1.0 & -0.5 & -1.0 \\
Speed & 0.5 & -0.5 & 0.5 & -0.5 & 0.5 \\
Liveliness & 0.5 & 1.0 & 0.5 & 1.0 & 0.5 \\
Transition & 0.5 & -0.5 & 0.5 & -0.5 & 0.5 \\
$\quad$ smoothness & & & & & \\
\hline
\end{tabular}

Given the limited nature of the data (three points for each set), for the correlation only $a+1$ or -1 result was taken as an indication of a strong association.

\section{Stability/accuracy/edge grip/speed/transition smoothness}

Interestingly, stability, accuracy, edge grip, speed and transition smoothness all possessed the same correlations with the key objective parameters. Whilst none of the relationships were strong, the values indicated that for a board with high levels of these subjective parameters, high bending/torsional stiffness in the body and camber is required. This was not surprising as high overall stiffness would promote a strong, stable and fast base for the rider (as indicated by the results of the highly stiff freeride board), as well as ensuring the board does not undergo drastic flex during turns, diminishing the grip.

Furthermore, the correlations suggested that a certain level of these objective parameters is required for a smooth transition between edges, as otherwise the board will feel limp and unresponsive, and the transition will be forced. Surprisingly however, relatively lower levels of heel and shovel stiffness weakly correlated to high levels of these performance parameters, which indicated that the results must be treated with caution. It was intuitively expected that the heel and shovel sections should have little influence on these factors, as they are not in contact with the snow for general riding.

\section{Manoeuvrability}

Manoeuvrability however provided some very interesting and somewhat surprising results. Whilst stability and manoeuvrability are polar opposites by definition, both the body stiffness parameters showed strong positive correlation (and the heel and shovel to a lesser extent) to the snowboard's manoeuvrability. It was expected that softness in the centre of the board would allow independent foot movement increasing rider control, yet evidence was provided to the contrary. The results could imply that as the stiffness in the snowboard increases, the transfer of forces from the rider to the board then the snow is quicker and more direct, increasing the overall manoeuvrability. The camber also showed a strong linear positive correlation to the manoeuvrability, which in combination with the previous results suggests that a stiff, highly cambered body section will allow the rider to swiftly generate turns by aggressively pushing the body of the snowboard into the snow (flattening the camber). These same properties would also allow a quick exit from a turn as the camber is returned to its natural position. 


\section{Feedback}

Given the previous recognition of feedback as the parameter with the highest level of subjectivity, any correlations had to be treated with caution. Yet the results were essentially as expected, with increasing stiffness along the entire chord and camber implying greater feedback to the rider. Interestingly, the heel and shovel stiffness provided the strongest positive association with feedback, possibly indicating the cantilever beam effect of the nose and tail greatly influences the rider's perception of feedback as the stiffness of these sections increases.

\section{Forgiveness}

Regarding forgiveness, as expected, the camber and stiffness showed medium to strong negative linear correlations, as a generally stiffer and more highly cambered board, whilst stable and accurate, would tend to punish any mistakes by the rider. Unlike the feedback results however, the body section appears to have a stronger connection with forgiveness than the heel and shovel regions, although these sections are used in the landing of tricks, where forgiveness is crucial.

\section{Board liveliness}

Interestingly, all objective parameter correlations with liveliness were positive, implying that increased stiffness and camber will promote a lively board. Whilst the camber result was unsurprising (although expected to be strong instead of medium), given the high liveliness of the most flexible freestyle board, it was perhaps expected that relatively low levels of body stiffness would lead to a more lively board. It was noted however that the response will highly depend on the mass and strength of the rider, as a heavier person may find a softer board less lively if the camber is too easily pressed, and consequently a stiffer board more suitable. Thus stiffness and camber levels must be tailored to individual riders in order to achieve an optimal response. The strong positive correlations for the heel and shovel were likely caused by the extra stiffness in these sections of the freestyle board, to ensure adequate spring for jumps and landings.

\section{Conclusion}

All of the test boards examined in this research possessed similar shaped bending and torsional profiles, comprising a steep rise in stiffness from the tip and tail towards the centre, yet all featured a substantial trough in the centre of the board. The similar bending curves were highly representative of each snowboard's respective thickness distribution. The torsional stiffness profiles however appear to be driven by the construction of the composite.

All of the subjective performance parameters except forgiveness showed positive associations to the body stiffness and camber, with manoeuvrability exhibiting the strongest correlations. The forgiveness showed the exact opposite trend, implying that higher levels of flex and less camber promotes a forgiving snowboard.

Overall, this analysis has provided further insight into the stiffness characteristics of snowboards and additionally, the correlation of key objective design features to subjective performance parameters. The information would help simplify tailoring the design of modern snowboards towards specific performance objectives.

Open Access This article is distributed under the terms of the Creative Commons Attribution Noncommercial License which permits any noncommercial use, distribution, and reproduction in any medium, provided the original author(s) and source are credited.

\section{References}

1. ASTM International (1995) Standard terminology relating to snowboarding, annual book of ASTM standards, F1107-F1995

2. Buckingham M, Blackford J (2004) Analysing snowboard mechanics. Snow engineering V-proceedings of the 5th international conference on snow engineering, 5-8 July 2004, Davos, Switzerland, pp 203-208

3. Buffinton K, Shooter S, Thorpe I, Krywicki J (2003) Laboratory, computational, and field study of snowboard dynamics. Sports Eng 6(3):129-137

4. Darques J, Carreira RP, de la Mettrie A, Bruyant D (2004) Quality function deployment-a means for developing adequate skis and snowboards. The engineering of sport 5-conference proceedings of the engineering of sport, 13-17 September 2004, Davis, California, pp 428-434

5. Federolf P, Auer M, Fauve M, Lüthi A, Rhyner H (2006) Subjective evaluation of the performance of Alpine Skis and correlations with mechanical ski properties. The engineering of sport 6-conference proceedings of the engineering of sport 11-14 July 2006, Munich, Germany, pp 287-292

6. International Standards Organization (1980) Alpine skis-determination of the elastic properties, ISO 5902:1980

7. Stevens R, Loudon D, Sherwood P, Dunn J (2006) Market opportunity analysis. Routledge, London, pp 129-138

8. Subic A, Clifton P, Beneyto-Ferre J (2008) Identification of innovation opportunities for snowboard design through benchmarking. Sports Technology, vol 1, no 1

9. Ulrich K, Eppinger S (2007) Product design and development. McGraw-Hill, Singapore, pp 37-43

10. Yang K, El-Haik B (2003) Design for six sigma. McGraw-Hill, New York, pp 184-186 